\title{
0 IJCRR \\ Section: Healthcare \\ Development of Analysis Model using Artificial Intelligence Algorithms for Post-Traumatic Stress Disorder Data: A Letter to the Editor
} ISI Impact Factor (2019-20): 1.628 IC Value (2019): 90.81 $\operatorname{SJIF}(2020)=7.893$

(c) (i) (3) Copyright@IJCRR

\author{
Harish Kumar Banga \\ Department of Mechanical Engineering, Guru Nanak Dev Engineering College, Ludhiana, Punjab India. \\ E-mail:drhkbanga@gmail.com
}

It is a pleasure writing a Letter to the Editor for the published article: Kim JB. A Study on the Development of Analysis Model using Artificial Intelligence Algorithms for PTSD (Post-Traumatic Stress Disorder) Data. Int J Cur Res Rev. 2020;12(16):60-65.

Although similar work has been done before in many health care institutes elsewhere, data indeed differs from centre to centre. Hence, it is a bold step not to become intimidated that it may be a repetition of previous work. It provides the ground reality in our country which we can refer to. Posttraumatic stress disorder (PTSD) is a psychiatric condition that leads to significant disability and impairment. Early interventions administered shortly after a traumatic event can reduce the onset of PTSD and associated long-term impairment. Given the costs associated with early intervention, it is not feasible or necessary to intervene with everyone exposed to these events rather, a screen-and-treat approach is recommended in which those at high risk for PTSD are identified and treated. A key barrier to providing early intervention is an inability to accurately identify those at high risk for PTSD in this acute post-trauma period $(<30$ days following an event). The limited ability to detect those at risk stems from a limited understanding of how PTSD symptoms develop, and thus, what factors are most helpful in determining risk for the disorder.

This research used the SVM (Support Vector Machine) analysis model and NNM (Neural Network Model) analysis model. The validation of the result is lacking in terms of the previous studies carried out in this field.

A diagnosis of PTSD requires symptoms to be present for at least 30 days. Previous studies suggest that symptoms first appear in the days and weeks after a traumatic event and gradually increase over time. Therefore, it may be possible to identify those at risk for PTSD by monitoring the progression of symptoms during this early period. Other previous studies have shown that effective monitoring and data collection can be implemented via a Smartphone survey. We hypothesized that predictive models based on statistical correlations between observable symptoms shortly after a traumatic event and eventual PTSD symptomology can be developed. Such predictive models would allow individuals at elevated risk for more severe psychopathology to be identified and provided with early intervention.

This study can be a starting point for the main purpose of the research is to develop intelligent analysis tools based on big data by the existing PTSD diagnostic criteria and implement analysis algorithms. The limitations of the methods of diagnosing and investigating these complex factors do not eliminate the possibility of contamination of the resulting data by the self-defense instinct of the trauma experience. The focus was on R\&D on the big data-based analysis system implementation algorithm that can identify and predict major risk factors of post-traumatic stress disorder (PTSD).

In the new study, the research required The Trauma History Questionnaire (1 month) to evaluate lifetime exposure to traumatic events. The Trauma History Questionnaire queries exposure to each of 13 distinct traumatic events, and for each event, the presence of fear, helplessness, or horror (as per DSM-IV PTSD Criterion A: A traumatic event). Two dichotomous variables are (a) exposure to childhood trauma and (b) exposure to adult life trauma.

Furthermore, data to be collected in adults, psychological and ecological risk factors known to be associated with PTSD include a history of extremely adverse life events, socio-demographic variables, social support factors, history of psychopathology, and environmental factors. Several studies have shown that childhood physical abuse, neglect, and sexual abuse are associated with the development of PTSD in adulthood.

The study should not end by stating data only, but identifying where the problems are present and take remedial meas- 
ures to reduce them. These early life events may contribute to an increased level of emotional and physiological arousal which has been associated with the development of PTSD in response to an event later in life. Pre-existing socio-demographic factors and later life events for the development of PTSD include female gender, non-white race/ethnicity, lower IQ and educational attainment, unemployment, history of incarceration, intimate partner physical abuse, and history of suicide.

There is increasing evidence that neighborhood-level and community-level factors play a role in risk for PTSD. These factors may interact with individual-level factors to comprise an individual's risk profile. Living close to a natural disaster or terrorist attack may increase an individual's risk for developing PTSD following the event. It has also been shown that exposure to community violence in childhood is a risk factor for developing PTSD later in life in youth living in both urban and suburban neighborhoods.

This research can also be improved by using the speech analysis technology used in the current study on PTSD detection falls into the range of capabilities included in our speech analytics platform called SenSay Analytics which give more effective and accurate results in the future.

I would like to thank the authors for their efforts to highlight the mental health damage factors for firefighters exposed to various trauma such as accidents, disasters, and stress because data on the subject in this country are few. This study should be the starting point for further studies based on AI algorithms and big data, this study aims to develop the analysis model about the prevalence of post-traumatic stress disorder. 